\title{
Asymptotic behavior of $N$-fields Chiral cosmology
}

\author{
Andronikos Paliathanasis ${ }^{1,2, a}$, Genly Leon $^{3, b}$ (B) \\ ${ }^{1}$ Instituto de Ciencias Físicas y Matemáticas, Universidad Austral de Chile, Valdivia, Chile \\ 2 Institute of Systems Science, Durban University of Technology, PO Box 1334, Durban 4000, South Africa \\ ${ }^{3}$ Departamento de Matemáticas, Universidad Católica del Norte, Avda. Angamos 0610, Casilla 1280, Antofagasta, Chile
}

Received: 27 July 2020 / Accepted: 28 August 2020 / Published online: 14 September 2020

(C) The Author(s) 2020

\begin{abstract}
We perform a detailed analysis for the asymptotic behaviour for the multi-scalar field Chiral cosmological scenario. We present the asymptotic behaviour for the one-field, two-fields and three-fields Chiral models. From these results, and deriving conserved quantities, we present a Theorem for the $N$-fields model for the Chiral model with $N$-fields. We find that the maximum number of scalar fields which provide interesting physical results at the background level is twofields, while for $N>2$ the new stationary points are only of mathematical interest since they do not describe new exact solutions different from those recovered for $N=2$.
\end{abstract}

\section{Introduction}

The detailed analysis of the recent cosmological observations indicated that our universe has gone through two acceleration phases [1-7]. A late-time acceleration phase [8] and an early acceleration phase known as inflation $[9,10]$. The main mechanics for the description of the inflation era is based on a scalar field, called inflation [11-14], which dominates in the early universe and drives into the current acceleration epoch. Moreover, the cause of the late-time acceleration is also unknown. The late-time acceleration it is attributed to a matter source called Dark-Energy.

Dark energy would need to have a strong negative pressure (repulsive action), to explain the observed acceleration of the expansion of the universe. In the Friedmann-LemaitreRobertson-Walker metric, it can be shown that a strong constant negative pressure in all the universe causes an acceleration in the expansion if the universe is already expanding, or a deceleration in contraction if the universe is already contracting. This accelerating expansion effect is sometimes labeled "gravitational repulsion". There are various attempts by the cosmologists to determine the physics behind the Dark

\footnotetext{
a e-mail: anpaliat@phys.uoa.gr

b e-mail: genly.leon@ucn.cl (corresponding author)
}

Energy. The main approaches are based on the introduction of scalar fields in the gravitational field equations [15-19], or are based on the introduction of other geometrodynamical terms which follow from the modification of the Einstein-Hilbert Action [20,21]. In the latter modified theories of gravity, the geometrodynamical terms can be described by scalar fields by attributing the new degrees of freedom. For an extended discussion we refer the reader to [22].

The simplest scalar field which has been introduced in the cosmological studies is the quintessence field [15], next it was introduced the phantom field or the k-essence theory $[18,19]$. Other scalar field models which have been proposed in the literature are nonminimally coupled with gravity, such as the Brans-Dicke theory [23], which belongs to the scalar tensor theories [24,25], or the more general Horndenski Action Integral [26] or its special cases like Galileon or cubic gravity [27-32]. Furthermore, it was introduced the teleparallel analog of Horndeski gravity [33-35], and $f\left(T, T_{G}\right)$ class of gravitational modification, based on the quadratic torsion scalar $T$, as well as on the new quartic torsion scalar $T_{G}$ which is the teleparallel equivalent of the Gauss-Bonnet term [36,37].

In order to overpass various problems in the description of the universe, multi-field cosmological models have been proposed. For instance, the two scalar field model known as quintom model consists of a quintessence and one phantom field [38]. In the quintom model, the parameter for the equation of state of the effective cosmological fluid can cross the phantom divide line more than once [38-43], without the existence of ghosts. Furthermore, multi-field models have been proposed to describe different mechanisms for the inflationary era [44-46]. Other multi-field models have been used to describe the "dark sector" of the universe, that is, the dark energy and the dark matter [47-51]. The dynamical system analysis at background and perturbation levels of $\Lambda \mathrm{CDM}$ and/or a quintessence field model with exponential potential was performed in [52-54], perturbations for interacting 
quintessence were studied in [55]. Perturbations of the Quintom Models of Dark Energy and the effects on observations were studied in [56], and for multi-field models in [57].

In [58] the dynamics of a multi-scalar field cosmology model was studied for a model which consists of various quintessence fields with independent scalar field potential. In this study, there was found that the model provides a new dynamical behaviour with physical importance. However, a questions which follows is: which is the number of scalars fields in the multi-field theory which provides results of physical interests.

In this work, we are interested in the multi-field theory known as Chiral model [59,60]. In particular we study the asymptotic behaviour of this multi-field model and we attempt to answer the questions of what the maximum number of fields is, and when we get the results of physical interests from. The model of our consideration is the $N$-field model proposed in [61] and generalizes the two-scalar Chiral model [62]. In particular, the Lagrangian function for the $N$-fields is considered to have a nonlinear kinetic part, such that the kinetic quantities of the fields would define the $N$-dimensional manifold with a maximally symmetric line element of constant nonzero curvature. For this specific theory and for various families of scalar field potentials, exact and analytic solutions have been determined [61].

In reference [63] it was generalized the Superpotential method for multi-field Chiral cosmological models with an arbitrary potential. The reduction of two-field models to single-field models can be performed by choosing the components of the metric tensor of a target (fields) space. The method of construction of Chiral cosmological models admitting exact solutions was developed. New classes of exact solutions in the two-field model connected with an $f(R)$ gravity model with an additional scalar field have been constructed, including modified power-law solutions.

The plan of the paper is as follows. In Sect. 2, we present the two-scalar field Chiral model where we discuss the main properties of the model and we show how the fields can interact. The $N$-field Chiral model is discussed in Sect. 3. The study for asymptotic behaviour of the cosmological field equations for a spatially flat FLRW background space is given in Sect. 4. More precisely, we present in detail the analysis for the asymptotic behaviour for the one-scalar field model, that is, the quintessence model, the two-scalar fields Chiral model, the three-scalar fields Chiral model, from where we observe that when we pass from one to two fields, new stationary points which describe different eras in the cosmological history exist. Moreover, when we pass from the two-field to the three-field model, we observe that new stationary points are obtained, but without new physical interests. In the two- and three-scalar field models, the only possible attractors are those of the quintessence theory, which is an interesting result because these multi-field models can be used either to describe the matter era if the model will be considered as a unification of the dark-energy and dark matter model, or by being considered as an inflationary model with two different behaviours in the inflationary era. As far as the $N$-fields model is concerned, the asymptotic behaviour is described by a theorem, from where we conclude that the main points of special physical interests at the background level are found for $N=2$, and the models with $N>2$ have only mathematical interests. Finally, in Sect. 5, we draw our conclusions.

\section{Chiral cosmology}

In Chiral model the gravitational Action Integral consists of two scalar fields [62]

$$
\begin{aligned}
S= & \int d x^{4} \sqrt{-g}\left(R-\frac{1}{2} g^{\mu \nu} \nabla_{\mu} \phi \nabla_{\nu} \phi\right. \\
& \left.-\frac{1}{2} g^{\mu \nu} e^{2 \sigma \phi} \nabla_{\mu} \psi \nabla_{\nu} \psi-V(\phi, \psi)\right),
\end{aligned}
$$

where $g_{\mu v}$ is the metric tensor of the underlying fourdimensional spacetime with Ricciscalar $R$ and $\phi=\phi\left(x^{\mu}\right)$, $\psi=\psi\left(x^{\mu}\right)$ are the two scalar fields minimally coupled to gravity but with interaction terms which depend on the coupling constant $\sigma$, while function $V(\phi, \psi)$ is the scalar field potential. We observe that the kinetic part of the two fields $\{\phi, \psi\}$ is defined by a space of constant curvature.

The Action Integral (1) can be seen as the generalization of a complex scalar field model with Action Integral

$S=\int d x^{4} \sqrt{-g}\left(R-\frac{1}{2} g^{\mu \nu} \nabla_{\mu} \Phi \nabla_{\nu} \Phi^{*}-V\left(\Phi, \Phi^{*}\right)\right)$

in which $\Phi=\phi+i \psi$ and the norm $|\Phi|^{2}=\Phi \Phi^{*}$ is defined as

$|\Phi|^{2}=\phi^{2}+e^{2 \sigma \phi} \psi^{2}$

For the background space we consider a spatially flat FLRW spacetime which is described by the line element

$d s^{2}=-d t^{2}+a^{2}(t)\left(d x^{2}+d y^{2}+d z^{2}\right)$,

where $a(t)$ is the scale factor and $H=\frac{\dot{a}}{a}$ is the Hubble function. The FLRW spacetime admits a six dimensional Killing algebra, which we assume that it is inherited by the two scalar fields $\{\phi, \psi\}$, that is $\phi=\phi(t)$ and $\psi=\psi(t)$. 
By replacing the Ricciscalar of the line element (4) in (1) the gravitational field equations follows [64]

$3 H^{2}=\frac{1}{2}\left(\dot{\phi}^{2}+e^{2 \sigma \phi} \dot{\psi}^{2}\right)+V(\phi, \psi)$,

$2 \dot{H}+3 H^{2}=-\left(\frac{1}{2}\left(\dot{\phi}^{2}+e^{2 \sigma \phi} \dot{\psi}^{2}\right)-V(\phi, \psi)\right)$,

while the scalar fields satisfy the continuity equations

$$
\begin{aligned}
& \ddot{\phi}+3 H \dot{\phi}-\sigma e^{2 \sigma \phi} \dot{\psi}^{2}+V_{, \phi}=0, \\
& \ddot{\psi}+3 H \dot{\psi}+2 \sigma \dot{\phi} \dot{\psi}+e^{-2 \sigma \phi} V_{, \psi}=0 .
\end{aligned}
$$

The field Eqs. (5)-(8) have been widely studied in the literature. Some exact solutions are presented in [62] while functional forms for the scalar field potential for which the field equations form a Liouville integrable system were determined in [64]. Scaling attractors in Chiral model were found in [65] and a detailed analysis of the dynamics of Chiral model was presented in [66]. Moreover in [67] extensions of the Chiral Action Integral (1) were studied.

However this is not the only parametrization for the Chiral model. Indeed without loss of generality we can define the new fields $\Phi, \Psi$ such that the Action Integral to be [64]

$$
\begin{aligned}
S= & \int d x^{4} \sqrt{-g}\left(R-\frac{1}{2} g^{\mu \nu} \nabla_{\mu} \Phi \nabla_{\nu} \Phi\right. \\
& \left.-\frac{1}{2} g^{\mu \nu} \sinh ^{2}(\kappa \Phi) \nabla_{\mu} \Psi \nabla_{\nu} \Psi-V(\Phi, \Psi)\right) .
\end{aligned}
$$

There is a one to one relation between the fields $\{\phi, \psi\} \rightarrow$ $\{\Phi, \Psi\}$, the two Action integrals (1), (9) are related under the action of a point transformation on the space of constant curvature for the scalar fields. In the following we are interested on the parametrization of the Action Integral (9).

For mass-free scalar fields $\{\Phi, \Psi\}$, and in the presence of the cosmological constant term, it was found in [68] that the equation of state parameter for the effective fluid can cross the phantom divide line as an effect of quantum transitions.

\section{$3 \mathrm{~N}$-field Chiral model}

A multi-field generalization of the Chiral model it was proposed in [61]. In particular we consider the multi-field Action Integral [61]

$$
S=\int \sqrt{-g} d x^{4} R-\int \sqrt{-g} d x^{4} L_{\Phi}\left(\Phi^{C}, \nabla_{\mu} \Phi^{C}\right),
$$

where $L_{\Phi}\left(\Phi^{C}, \nabla_{\mu} \Phi^{C}\right)$ is the Action integral for the $N$ fields $\Phi^{A}=\left(\phi_{1}, \phi_{2}, \ldots, \phi_{N}\right)$ given as follows [61]

$$
\begin{aligned}
& L_{\Phi}\left(\Phi^{C}, \nabla_{\mu} \Phi^{C}\right) \\
& \quad=\frac{1}{2} g^{\mu \nu} \Xi_{A B}\left(\Phi^{C}\right) \nabla_{\mu} \Phi^{A} \nabla_{\nu} \Phi^{B}+V\left(\Phi^{C}\right),
\end{aligned}
$$

were $\Xi_{A B}\left(\Phi^{C}\right)$ is a second-rank tensor and defines the space where the scalar fields evolves. For the quintom model, $\Xi_{A B}\left(\Phi^{C}\right)$ is a two-dimensional flat space of Lorentzian signature, while for the Chiral model $\Xi_{A B}\left(\Phi^{C}\right)$ is a twodimensional space of constant (negative) curvature, for instance

$\Xi_{A B}\left(\Phi^{C}\right)=\operatorname{diag}\left(1, \sinh ^{2}\left(\kappa \phi_{1}\right)\right)$.

Therefore, in order to consider a $N$-field extension of the Chiral model we assume that $\Xi_{A B}\left(\Phi^{C}\right)$ is a $N$-dimensional space of constant curvature, that is, [61]

$$
\begin{aligned}
\Xi_{A B}\left(\Phi^{C}\right) \\
=\operatorname{diag}\left(1, \sinh ^{2}\left(\kappa \phi_{1}\right), \sinh ^{2}\left(\kappa \phi_{1}\right) \sin ^{2}\left(\kappa \phi_{2}\right),\right. \\
\left.\quad \ldots, \sinh ^{2}\left(\kappa \phi_{1}\right) \sin ^{2}\left(\kappa \phi_{2}\right) \ldots \sin ^{2}\left(\kappa \phi_{N-1}\right)\right) .
\end{aligned}
$$

For the line element (10) with Lagrangian density (11) the gravitational field equations are

$G_{\mu \nu}=T_{\mu \nu}\left(\Phi^{C}, \nabla_{\mu} \Phi^{C}\right)$,

in which

$$
\begin{aligned}
& T_{\mu \nu}\left(\Phi^{C}, \nabla_{\mu} \Phi^{C}\right)=\Xi_{A B}\left(\Phi^{C}\right) \nabla_{\mu} \Phi^{A} \nabla_{\nu} \Phi^{B} \\
& \quad-g_{\mu \nu}\left(-\frac{1}{2} g^{\mu \nu} \Xi_{A B}\left(\Phi^{C}\right) \nabla_{\mu} \Phi^{A} \nabla_{\nu} \Phi^{B}-V\left(\Phi^{C}\right)\right),
\end{aligned}
$$

while the equation of motions for the scalar fields are given by the $N$-dimensional vector field

$g^{\mu \nu}\left(\nabla_{\mu} \Xi_{B}^{A}\left(\Phi^{C}\right) \nabla_{\nu} \Phi^{B}\right)+\Xi_{B}^{A}\left(\Phi^{C}\right) \frac{\partial V\left(\Phi^{C}\right)}{\partial \Phi^{B}}=0$.

For the cosmological line element (4) the Friedmann equations reads

$$
\begin{aligned}
& -3 H^{2}+\frac{1}{2} \Xi_{A B}\left(\Phi^{C}\right) \dot{\Phi}^{A} \dot{\Phi}^{B}+V\left(\Phi^{C}\right)=0 \\
& -2 \dot{H}-3 H^{2}+\frac{1}{2} \Xi_{A B}\left(\Phi^{C}\right) \dot{\Phi}^{A} \dot{\Phi}^{B}-V\left(\Phi^{C}\right)=0,
\end{aligned}
$$

and the conservation laws are

$\ddot{\Phi}^{A}+\tilde{\Gamma}_{B C}^{A}\left(\Phi^{D}\right) \dot{\Phi}^{B} \dot{\Phi}^{C}+3 H \dot{\Phi}^{A}+\Xi^{A B}\left(\Phi^{C}\right) V_{, B}\left(\Phi^{C}\right)=0$, 
where $\tilde{\Gamma}_{B C}^{A}\left(\Phi^{D}\right)$ denotes the Levi-Civita connection coefficients of $\Xi_{A B}\left(\Phi^{C}\right)$.

\section{Asymptotic behaviour}

In this section we study the effects of the additional scalar fields in the asymptotic behaviour of the cosmological solution. In order to perform this analysis we start our presentation by assuming one scalar field model $\Phi^{A}=\phi$, which correspond to the quintessence model. For the scalar field potential we consider the exponential potential $V\left(\Phi^{A}\right)=V_{0} e^{\lambda \phi}$.

In order to study the asymptotic behaviour of the multifield model, we define the dimensionless variables [69]

$$
\begin{aligned}
x_{1}= & \frac{\dot{\phi}_{1}}{\sqrt{6} H}, \\
x_{2}= & \sinh \left(\kappa \phi_{1}\right) \frac{\dot{\phi}_{2}}{\sqrt{6} H}, \\
x_{3}= & \sinh \left(\kappa \phi_{1}\right) \sin \left(\kappa \phi_{2}\right) \frac{\dot{\phi}_{3}}{\sqrt{6} H}, \\
& \ldots \\
x_{N}= & \sinh \left(\kappa \phi_{1}\right) \sin \left(\kappa \phi_{2}\right) \ldots \sin \left(\kappa \phi_{N-1}\right) \frac{\dot{\phi}_{N}}{\sqrt{6} H},
\end{aligned}
$$

and

$y=\sqrt{\frac{V\left(\phi_{1}\right)}{3 H^{2}}}$,

where the Friedmann equation reads

$1-y^{2}-\left(\left(x_{1}\right)^{2}+\left(x_{2}\right)^{2}+\cdots+\left(x_{N}\right)^{2}\right)=0$

while the equation of state parameter for the effective fluid becomes

$w_{e f f}=\left(x_{1}\right)^{2}+\left(x_{2}\right)^{2}+\ldots+\left(x_{N}\right)^{2}-y^{2}$.

\subsection{Quintessence}

For the quintessence model, that is, for one scalar field model, the gravitational field equations are written in the form of the following system [69]

$$
\begin{aligned}
& \frac{d x_{1}}{d \tau}=\frac{1}{2}\left(3 x_{1}^{3}-3 x_{1}\left(1+y^{2}\right)-\sqrt{6} \lambda y^{2}\right), \\
& \frac{d y}{d \tau}=\frac{1}{2} y\left(3\left(1+x_{1}^{2}-y^{2}\right)+\sqrt{6} \lambda x_{1}\right) .
\end{aligned}
$$

where we have selected the new independent variable to be $\tau=\ln a$.
The stationary points $P=\left(x_{1}, y\right)$ of the system (22), (23) which satisfy the constraint (20) are [69]

$$
P_{1}^{( \pm)}=( \pm 1,0), P_{2}=\left(-\frac{\lambda}{\sqrt{6}}, \sqrt{1-\frac{\lambda^{2}}{6}}\right) \text {. }
$$

The parameter for the equation of state for the effective fluid at the stationary points are $w_{\text {eff }}\left(P_{1}^{( \pm)}\right)=1$ and $w_{\text {eff }}\left(P_{2}\right)=-1+\frac{\lambda^{2}}{3}$. The exact solutions at the stationary points $P_{1}^{( \pm)}$describe universes where only the kinetic part of the scalar field dominates and the exact solution is that of the stiff fluid source. On the other hand, at the stationary point $P_{2}$ the scale factor describes a scaling solution and acceleration occurs when $|\lambda|<\sqrt{2}$. Point $P_{2}$ describes a de Sitter universe only when $\lambda=0$, which means that the scalar field potential plays the role of the cosmological constant, while point $P_{2}$ is physically accepted when $|\lambda|<\sqrt{6}$.

In order to study the stability of the stationary points we use the constraint Eq. (20) to reduce the dynamical system (22), (23) into the one dimensional equation [69]

$\frac{d x_{1}}{d \tau}=3\left(x^{2}-1\right)\left(x+\frac{\lambda}{\sqrt{6}}\right)$

from where we find that eigenvalues the linearized system at the stationary points are

$e\left(P_{1}^{ \pm}\right)=\sqrt{6} \lambda \pm 6, e\left(P_{2}\right)=\frac{1}{2}\left(\lambda^{2}-6\right)$.

We conclude that point $P_{1}^{+}$is an attractor when $\lambda<-\sqrt{6}$, point $P_{1}^{-}$is an attractor when $\lambda>\sqrt{6}$ while $P_{2}$ is always an attractor [69]. We continue with the two-scalar field model.

\subsection{Two-field model}

For the two scalar fields model, $\Phi^{A}=\left(\phi_{1}, \phi_{2}\right)$, we consider the exponential dependence on the first scalar field, $V(\Phi)=$ $V_{0} e^{\lambda \phi_{1}}$, for simplicity. Notice there is a nontrivial interaction between the scalar fields which follows from the nonlinear terms of the scalar field Lagrangian function.

The field equations in the dimensionless variables read

$$
\begin{aligned}
\frac{d x_{1}}{d \tau} & =\frac{1}{2}\left(3 x_{1}^{3}-3 x_{1}\left(1+y^{2}-x_{2}^{2}\right)-\sqrt{6} \lambda y^{2}+\sqrt{6} \mu_{1} x_{2}^{2}\right) \\
\frac{d x_{2}}{d \tau} & =\frac{1}{2} x_{2}\left(3\left(x_{1}^{2}+x_{2}^{2}-y^{2}-1\right)-\sqrt{6} \mu_{1} x_{1}\right) \\
\frac{d y}{d \tau} & =\frac{1}{2} y\left(3\left(1+x_{1}^{2}+x_{2}^{2}-y^{2}\right)+\sqrt{6} \lambda x_{1}\right) \\
\frac{d \mu_{1}}{d \tau} & =\sqrt{\frac{3}{2}} x_{1}\left(4 \kappa^{2}-\mu_{1}^{2}\right)
\end{aligned}
$$

where the variable $\mu_{1}$ is defined as $\mu_{1}=2 \kappa \operatorname{coth}\left(\kappa \phi_{1}\right)$. 
The stationary points $\bar{P}=\left(x_{1}, y, x_{2}, \mu_{1}\right)$ of the fourdimensional dynamical system (25)-(28) which satisfy the constraint condition (20) are

$$
\begin{aligned}
& P_{1}^{( \pm, \pm)}=( \pm 1,0,0, \pm 2 \kappa), \\
& P_{2}^{( \pm)}=\left(-\frac{\lambda}{\sqrt{6}}, \sqrt{1-\frac{\lambda^{2}}{6}}, 0, \pm 2 \kappa\right), \\
& P_{3}^{( \pm)}=(0,0, \pm 1,0), \\
& P_{4}^{( \pm)}=\left(\frac{\sqrt{6}}{2 \kappa-\lambda}, \sqrt{\frac{2 \kappa}{2 \kappa-\lambda}}, \pm \sqrt{\frac{\lambda^{2}-2 \lambda \kappa-6}{(2 \kappa-\lambda)^{2}}},-2 \kappa\right), \\
& P_{5}^{( \pm)}=\left(-\frac{\sqrt{6}}{2 \kappa+\lambda}, \sqrt{\frac{2 \kappa}{2 \kappa+\lambda}}, \pm \sqrt{\frac{\lambda^{2}+2 \lambda \kappa-6}{(2 \kappa+\lambda)^{2}}}, 2 \kappa\right) .
\end{aligned}
$$

Points $P_{1}^{( \pm, \pm)}, \quad P_{2}^{( \pm)}$have the same physical properties as for $P_{1}^{( \pm)}$and $P_{2}$, for the quintessence model. In particular they can be seen as the extension of the stationary points for the quintessence in the four-dimensional manifold of the variables $\left(x_{1}, y, x_{2}, \mu_{1}\right)$.

Points $P_{3}^{( \pm)}$describe universes dominated by the kinetic term of the second scalar field, while the parameter for the equation of state for the effective fluid is $w_{\text {eff }}\left(P_{3}^{( \pm)}\right)=1$. Points $P_{4}^{( \pm)}$, are real and physically accepted when

$$
\{\kappa>0, \lambda \leq-\sqrt{6}\} \cup\left\{-\sqrt{6}<\lambda<0,2 \kappa \geq \frac{\lambda^{2}-6}{\lambda}\right\},
$$

while points $P_{5}^{( \pm)}$exist when $\{\kappa>0, \lambda>\sqrt{6}\}$ $\cup\left\{0<\lambda \leq \sqrt{6}, 2 \kappa>\frac{6-\lambda^{2}}{\lambda}\right\}$. The exact solutions at the critical points describe scaling solutions with equation of state parameter for the effective fluid

$w_{e f f}\left(P_{4}^{( \pm)}\right)=1+\frac{4 \kappa}{\lambda-2 \kappa}, w_{e f f}\left(P_{5}^{( \pm)}\right)=1-\frac{4 \kappa}{\lambda+2 \kappa}$,

from were we can infer that $w_{\text {eff }}\left(P_{4}^{( \pm)}\right)<-\frac{1}{3}$ for $\{\lambda \leq-\sqrt{2}, \kappa>-\lambda\} \cup\left\{-\sqrt{2}<\lambda<0,2 \kappa \geq \frac{\lambda^{2}-6}{\lambda}\right\}$ and $w_{\text {eff }}\left(P_{5}^{( \pm)}\right)<-\frac{1}{3}$ when $\{\kappa>\lambda, \lambda>\sqrt{2}\}$ $\cup\left\{0<\lambda<\sqrt{2}, 2 \kappa \geq \frac{\lambda^{2}-6}{\lambda}\right\}$. For the stability analysis we apply the constraint Eq. (20) to reduce the dimension of the dynamical system by one, in particular we replace $y=\sqrt{1-x_{1}^{2}-x_{2}^{2}}$.

The eigenvalues of the stationary points $P_{1}^{( \pm, \pm)}$are

$$
\begin{aligned}
& e_{1}\left(P_{1}^{( \pm, \pm)}\right)=\varepsilon \sqrt{6} \lambda+6, e_{2}\left(P_{1}^{( \pm, \pm)}\right)=-2 \varepsilon \sqrt{6} \kappa, \\
& e_{3}\left(P_{1}^{( \pm, \pm)}\right)=-\varepsilon \sqrt{6} \kappa, \varepsilon=( \pm 1)( \pm 1) .
\end{aligned}
$$

Hence, for $\varepsilon=+1$, the stationary points are attractors when $\kappa>0$ and $\lambda<-\sqrt{6}$, while for $\varepsilon=-1$, the stationary points are attractors for $\lambda>\sqrt{6}$ and $\kappa<0$.

For the stationary points $P_{2}^{( \pm)}$the eigenvalues are

$$
\begin{aligned}
& e_{1}\left(P_{2}^{( \pm)}\right)=\frac{1}{2}\left(\lambda^{2}-6\right), e_{2}\left(P_{2}^{( \pm)}\right)=2 \kappa \lambda, \\
& e_{3}\left(P_{2}^{( \pm)}\right)=\frac{1}{2}\left(\lambda^{2}+2 \kappa \lambda-6\right)
\end{aligned}
$$

which follows that $P_{2}^{( \pm)}$are attractors when $\{-\sqrt{6}<\lambda<0, \kappa>0\} \cup\{0<\lambda<\sqrt{6}, \kappa<0\}$.

For the stationary points $P_{3}^{( \pm)}$the stationary points are

$e_{1}\left(P_{3}^{( \pm)}\right)=6, e_{2}\left(P_{3}^{( \pm)}\right)=\sqrt{6} \kappa, e_{3}\left(P_{3}^{( \pm)}\right)=-\sqrt{6} \kappa$,

from where we infer that the points are always saddle points.

As far as the stationary points $P_{4}^{( \pm)}$are concerned it follows that one of the eigenvalues is $e_{1}\left(P_{4}^{( \pm)}\right)=\frac{12 \kappa}{2 \kappa-\lambda}$, which follows that it is always positive when $P_{4}^{( \pm)}$are real points, hence the exact solutions at these two points are always unstable. Similarly, for $P_{5}^{( \pm)}$one of the eigenvalues is derived to be $e_{1}\left(P_{5}^{( \pm)}\right)=\frac{12 \kappa}{2 \kappa+\lambda}$ which is always positive hence the two exact solutions at $P_{5}^{( \pm)}$are always unstable.

We observe that the existence of the second scalar field introduce new stationary points in the cosmological evolution which can describe various cosmological eras. In particular, the additional points $P_{3}^{( \pm)}, P_{4}^{( \pm)}$and $P_{5}^{( \pm)}$, where the second scalar field contributes, provide unstable exact solutions, while points $P_{2}^{( \pm)}$can be seen as the future attractors. However, the set of points $P_{4}^{( \pm)}, P_{5}^{( \pm)}$can describe either a matter dominated era, or the early acceleration phase.

If $w_{\text {eff }}\left(P_{4}^{( \pm)}\right)=0$, such that the point to describe the matter epoch, it follows $2 \kappa=-\lambda$ and the coordinates of the points are

${ }^{[m]} P_{4}^{( \pm)}=\left(-\sqrt{\frac{3}{2}} \frac{1}{\lambda}, \frac{1}{\sqrt{2}}, \pm \frac{\sqrt{2 \lambda^{2}-6}}{2 \lambda}, \lambda\right)$

with $|\lambda|>\sqrt{3}$. Similarly $w_{\text {eff }}\left(P_{5}^{( \pm)}\right)=0$ gives $2 \kappa=-\lambda$, and in that case ${ }^{[m]} P_{4}^{( \pm)}={ }^{[m]} P_{5}^{( \pm)}$.

We observe that in this two-scalar fields model we do not recover the results presented in $[65,67]$ and that is because we used a different reparametrization for the scalar fields, which means that the scalar fields have different interaction terms. We continue our analysis by considering three-scalar fields. 


\subsection{Three-field model}

For the three-scalar field model, $\Phi^{A}=\left(\phi_{1}, \phi_{2}, \phi_{3}\right)$, and, as before, the potential $V\left(\Phi^{A}\right)=V_{0} e^{\lambda \phi_{1}}$ the field equations in the dimensional variables are written

$$
\begin{aligned}
\frac{d x_{1}}{d \tau}= & \frac{1}{2}\left(3 x_{1}^{3}-3 x_{1}\left(1+y^{2}-x_{2}^{2}\right)\right. \\
& \left.-\sqrt{6} \lambda y^{2}+\sqrt{6} \mu_{1}\left(x_{2}^{2}+x_{3}^{2}\right)\right) \\
\frac{d x_{2}}{d \tau}= & \frac{1}{2} x_{2}\left(3\left(x_{1}^{2}+x_{2}^{2}+x_{3}^{2}-y^{2}-1\right)-\sqrt{6} \mu_{1} x_{1}\right) \\
& +\frac{\sqrt{6}}{2} \mu_{2} x_{3}^{2} \\
\frac{d x_{3}}{d \tau}= & \frac{1}{2} x_{3}\left(3\left(x_{1}^{2}+x_{2}^{2}+x_{3}^{2}-y^{2}-1\right)\right. \\
& \left.-\sqrt{6}\left(\mu_{1} x+\mu_{2} x_{2}\right)\right) \\
\frac{d y}{d \tau}= & \frac{1}{2} y\left(3\left(1+x_{1}^{2}+x_{2}^{2}+x_{3}^{2}-y^{2}\right)+\sqrt{6} \lambda x_{1}\right) \\
\frac{d \mu_{1}}{d \tau}= & \sqrt{\frac{3}{2}} x_{1}\left(4 \kappa^{2}-\mu_{1}^{2}\right) \\
\frac{d \mu_{2}}{d \tau}= & \sqrt{\frac{3}{2}}\left(4 \kappa^{2} x_{2}-\mu_{1} \mu_{2} x_{1}-x_{2}\left(\mu_{1}^{2}+3 \mu_{2}\right)\right. \\
& \left.+\sqrt{\frac{3}{2}} \frac{\left(\mu_{1} \mu_{2}\right)^{2}}{4 \kappa^{2}} x_{2}\right)
\end{aligned}
$$

where $\mu_{2}=2 \kappa \frac{\cot \kappa \phi_{2}}{\sinh \kappa \phi_{1}}$. The stationary points $P=\left(x_{1}, y, x_{2}, \mu_{1}, x_{3}, \mu_{2}\right)$ for the latter dynamical system are

$$
\begin{aligned}
& P_{1}^{( \pm, \pm)}=( \pm 1,0,0, \pm 2 \kappa, 0,0), \\
& P_{2}^{( \pm)}=\left(-\frac{\lambda}{\sqrt{6}}, \sqrt{1-\frac{\lambda^{2}}{6}}, 0, \pm 2 \kappa, 0,0\right) \\
& P_{3}^{( \pm, \pm)}=\left(0,0, \pm 1,0,0, \pm \frac{2 \sqrt{3} \kappa}{3}\right) \\
& P_{4}^{( \pm, \pm)}=\left(\frac{\sqrt{6}}{2 \kappa-\lambda}, \sqrt{\frac{2 \kappa}{2 \kappa-\lambda}}, \pm \sqrt{\frac{\lambda^{2}-2 \lambda \kappa-6}{(2 \kappa-\lambda)^{2}}},\right. \\
& \left.-2 \kappa, 0, \pm \frac{\sqrt{6} \kappa}{\sqrt{\lambda^{2}+2 \kappa \lambda-6}}\right) \text {, } \\
& P_{5}^{( \pm, \pm)}=\left(-\frac{\sqrt{6}}{2 \kappa+\lambda}, \sqrt{\frac{2 \kappa}{2 \kappa+\lambda}}, \pm \sqrt{\frac{\lambda^{2}+2 \lambda \kappa-6}{(2 \kappa+\lambda)^{2}}},\right. \\
& \left.2 \kappa, 0, \pm \frac{\sqrt{6} \kappa}{\sqrt{\lambda^{2}+2 \kappa \lambda-6}}\right), \\
& P_{6}^{( \pm)}=(0,0,0,0, \pm 1,0) \text {, }
\end{aligned}
$$

$$
\begin{aligned}
P_{7}^{( \pm)}= & \left(\frac{\sqrt{6}}{2 \kappa-\lambda}, \sqrt{\frac{2 \kappa}{2 \kappa-\lambda}}, x_{2},-2 \kappa,\right. \\
& \left. \pm \sqrt{\frac{(\lambda-2 \kappa)\left(\lambda-x_{2}(\lambda-2 \kappa)\right)-6}{(\lambda-2 \kappa)^{2}}}, 0\right), \\
P_{8}^{( \pm)}= & \left(-\frac{\sqrt{6}}{2 \kappa-\lambda}, \sqrt{\frac{2 \kappa}{2 \kappa-\lambda}}, x_{2}, 2 \kappa,\right. \\
& \left. \pm \sqrt{\frac{(\lambda-2 \kappa)\left(\lambda-x_{2}(\lambda-2 \kappa)\right)-6}{(\lambda-2 \kappa)^{2}}}, 0\right) .
\end{aligned}
$$

Points $P_{1}^{( \pm, \pm)}, P_{2}^{( \pm)}, P_{3}^{( \pm, \pm)}$, are the extensions of the stationary points for the two fields in the six-dimensional space, while the new stationary points are $P_{4}^{( \pm, \pm)}, P_{5}^{( \pm, \pm)}, P_{6}^{( \pm)}, P_{7}^{( \pm)}$and $P_{8}^{( \pm)}$. Points $P_{7}^{( \pm)}, P_{8}^{( \pm)}$ are actually surfaces in the space $\left\{x_{2}, x_{3}\right\}$, while when $x_{2}=$ $\pm \sqrt{\frac{\lambda^{2}-2 \lambda \kappa-6}{(2 \kappa-\lambda)^{2}}}$ the points reduce to that of $P_{4}^{( \pm)}, P_{5}^{( \pm)}$for the two-scalar fields model.

The physical properties of points $P_{1}^{( \pm, \pm)}, P_{2}^{( \pm)}, P_{3}^{( \pm, \pm)}$ are the same as for the two fields case; $P_{6}^{( \pm)}$describe universes dominated by the kinetic part for the second scalar field, and the parameter for the equation of state is calculated $w_{\text {eff }}\left(P_{6}^{( \pm)}\right)=1$. Furthermore, the physical solutions at the stationary points $P_{4}^{( \pm, \pm)}, P_{5}^{( \pm, \pm)}, P_{7}^{( \pm)}$and $P_{8}^{( \pm)}$are the same with that of points $P_{4}^{( \pm)}$and $P_{5}^{( \pm)}$.

Consequently, the introduction of the new scalar field provides families of new stationary points but with not new physical properties. We proceed with the discussion on the stability of the exact solutions at the critical points.

As far as the stability properties of the points $P_{1}^{( \pm, \pm)}, P_{2}^{( \pm)}, P_{3}^{( \pm, \pm)}$are concerned, points $P_{3}^{( \pm, \pm)}$are always saddle points, while $P_{1}^{( \pm, \pm)}, P_{2}^{( \pm)}$can be attractors for the same values of the free parameters as for the twoscalar field model. The eigenvalues of the linearized system around the points $P_{6}^{( \pm)}$are derived

$$
\begin{aligned}
& e_{1}\left(P_{6}^{( \pm)}\right)=\frac{3}{2}, e_{2,3}\left(P_{6}^{( \pm)}\right)=-\frac{3+\sqrt{3\left(3+32 \kappa^{2}\right)}}{4}, \\
& e_{4,5}\left(P_{6}^{( \pm)}\right)=-\frac{3-\sqrt{3\left(3+32 \kappa^{2}\right)}}{4}
\end{aligned}
$$

from which we conclude that the points are saddle. Finally, for the rest sets of points, namely, $P_{4}^{( \pm, \pm)}, P_{5}^{( \pm, \pm)}, P_{7}^{( \pm)}$ and $P_{8}^{( \pm)}$at least of one of the eigenvalues has the value $e_{1}\left(P_{4}^{( \pm)}\right)=\frac{12 \kappa}{2 \kappa-\lambda}$ or $e_{1}\left(P_{5}^{( \pm)}\right)=\frac{12 \kappa}{2 \kappa+\lambda}$ from which we can infer that the exact solutions at the stationary points are always unstable, while the points are found to be saddle. Therefore the only future attractors can be the exact solutions of the two-scalar fields model, namely points $P_{1}^{( \pm, \pm)}, P_{2}^{( \pm)}$. 


\section{$4.4 N$-field model}

From the given analysis we see that the two-scalar field models provide new physical solutions in comparison with the quintessence model. On the other hand, in the three-scalar field model the new stationary points do not describe new physical universes, while the only attractors are the exact solution of the quintessence field. In order to understand that let us study the invariant surfaces of the multi-field model.

The point-like Lagrangian of the $N$-fields model reads

$$
\begin{aligned}
& L(a, \dot{a}, \Phi, \dot{\Phi})=-V_{0} a^{3} e^{\lambda \phi_{1}}-3 a \dot{a}^{2} \\
& \quad+\frac{1}{2} a^{3}\left(\dot{\phi}_{1}^{2}+\sinh ^{2}\left(\kappa \phi_{1}\right)\left(\dot{\phi}_{2}^{2}+\sin ^{2}\left(\kappa \phi_{2}\right)\left(\dot{\phi}_{3}^{2}+\cdots\right)\right)\right) .
\end{aligned}
$$

The latter point-like Lagrangian function is autonomous which means that admits as Noether symmetry [70] the vector field $\partial_{t}$ with conservation law the Hamiltonian function $\mathcal{H}(a, \dot{a}, \Phi, \dot{\Phi})=h$, which is nothing else than the constraint equation (17) which means that $\mathcal{H}(a, \dot{a}, \Phi, \dot{\Phi})=0$. Hence it follows

$$
\begin{aligned}
& \mathcal{H}(a, \dot{a}, \Phi, \dot{\Phi}) \equiv V_{0} a^{3} e^{\lambda \phi_{1}}-3 a \dot{a}^{2}+\frac{1}{2} a^{3} \\
& \left(\dot{\phi}_{1}^{2}+\sinh ^{2}\left(\kappa \phi_{1}\right)\left(\dot{\phi}_{2}^{2}+\sin ^{2}\left(\kappa \phi_{2}\right)\left(\dot{\phi}_{3}^{2}+\cdots\right)\right)\right)=0 .
\end{aligned}
$$

However, except from the Hamiltonian function, the dynamical system described by the point-like Lagrangian (35) admits additional conservation laws. Someone can apply Noether's theorem or other methods to construct conservation laws [71]. Indeed, one of conservation laws admitted by the dynamical system is the quadratic conservation law [72]

$$
I_{0}^{2}=a^{6} \sinh ^{4}\left(\kappa \phi_{1}\right)\left(\dot{\phi}_{2}^{2}+\sin ^{2}\left(\kappa \phi_{2}\right)\left(\dot{\phi}_{3}^{2}+\cdots\right)\right),
$$

where now the Hamiltonian function reads

$$
\begin{aligned}
\mathcal{H}(a, \dot{a}, \Phi, \dot{\Phi}) \equiv & -3 a \dot{a}^{2}+\frac{1}{2} a^{3}\left(\dot{\phi}_{1}^{2}+V_{0} a^{3} e^{\lambda \phi_{1}}\right) \\
& +\frac{I_{0}^{2}}{2 a^{3} \sinh ^{2}\left(\kappa \phi_{1}\right)} .
\end{aligned}
$$

For $I_{0}=0$, the dynamics reduces to that of quintessence field, however for $I_{0} \neq 0$ the new term drives the dynamics and provide the new additional physical behaviour. The quadratic conservation law $I_{0}$ defines an invariant surface of the dynamical system, while we observe that invariant subsurfaces exist also in $I_{0}$, however they do not affect the final dynamics at the background level.

Consequently, we have the following theorem.
Theorem For the Chiral model with $N$-fields, with $N>2$ the exact cosmological solutions which are described by the stationary points have the same physical properties of the two-scalar field model, $N=2$, at the background level, which means that the possible solutions are: the stiff fluid solution, scaling solution of quintessence and a scaling solution where two or more scalar fields contributes in the total cosmological fluid. Finally, the quintessence model is the future attractor of the $N$ - field Chiral model.

The above theorem says that in this Chiral theory, the consideration of more than two scalar fields is only of mathematical interests and there is not any new physical properties at the background level. The scalar fields are nonlinear and there are interaction terms which follow from the kinetic terms of the Lagrangian function. Of course the consideration of a more general potential function can lead to a different result, but again the limit of the quintessence field will always exists, and the above theorem will holds. Finally, by considering the analysis of the evolution of cosmological perturbations in multi-field cosmologies, the parameters of cosmological perturbations for different numbers of scalar fields will generally differ, see, for example, in [57]. However, our result holds at the level of background.

\section{Conclusions}

In this work we considered a multi-field cosmological model. Specifically, we assume the existence of $N$-fields in a spatially flat FLRW background space which interact in the kinetic terms. That model is the multi-field extension of the Chiral theory, where the dynamics of the fields are over an space of constant curvature.

We focus on the contribution of the new fields in the cosmological evolution. In particular we wanted to answer the question of how essential is the introduction of the new scalar fields in the cosmological dynamics. In order to work in this direction we focus on the asymptotic analysis for the dynamics of the cosmological field equations. We presented a detailed analysis of the stationary points for the one-field model, which is the quintessence with exponential scalar field, for the two-fields Chiral model and for the three-fields extension. From these results we found that when we pass from the one-field to the two-fields model, the cosmological behaviour becomes richer. New stationary points follows which provide new physical solutions.

However, when we pass from the two-fields to the threefields model, the new stationary points do not provide new physical solutions, while the additional stationary points are only of mathematical interests. For the $N$-fields model, and with the use of invariant functions, we describe the physical properties, and the asymptotic behaviour for the field 
equations in a theorem. The theorem states that the only possible physical solutions of the $N$-fields model that exists are those that describes: stiff fluid dominated universes, the quintessence scaling solution and a scaling solution where two or more scalar fields contributes in the total cosmological fluid.

From the above analysis we conclude that in this specific theory, the consideration of more than two scalar fields does not affect the physics at the level of background dynamics. Applications of cosmological perturbations of multi-field cosmological models, however, will provide more insight of the subject, but it is out of the scope of the present study.

Acknowledgements AP \& GL were funded by Agencia Nacional de Investigación y Desarrollo - ANID through the program FONDECYT Iniciación Grant no. 11180126. Additionally, GL is supported by Vicerrectoría de Investigación y Desarrollo Tecnológico at Universidad Católica del Norte.

Data Availability Statement This manuscript has no associated data or the data will not be deposited. [Authors' comment: This is a Theoretical Research Project].

Open Access This article is licensed under a Creative Commons Attribution 4.0 International License, which permits use, sharing, adaptation, distribution and reproduction in any medium or format, as long as you give appropriate credit to the original author(s) and the source, provide a link to the Creative Commons licence, and indicate if changes were made. The images or other third party material in this article are included in the article's Creative Commons licence, unless indicated otherwise in a credit line to the material. If material is not included in the article's Creative Commons licence and your intended use is not permitted by statutory regulation or exceeds the permitted use, you will need to obtain permission directly from the copyright holder. To view a copy of this licence, visit http://creativecomm ons.org/licenses/by/4.0/.

Funded by SCOAP ${ }^{3}$.

\section{References}

1. A.G. Riess et al., Astron. J. 116, 1009 (1998)

2. S. Perlmutter et al., Astrophys. J. 517, 565 (1998)

3. P. Astier et al., Astrophys. J. 659, 98 (2007)

4. N. Suzuki et al., Astrophys. J. 746, 85 (2012)

5. G. Hinshaw et al., [WMAP Collaboration], Astrophys. J. Suppl. 208, 19 (2013)

6. P.A.R. Ade et al., [Planck Collaboration], Astron. Astrophys. 594, A13 (2016)

7. N. Aghanim et al. [Planck Collaboration]. arXiv:1807.06209 [astro-ph.CO]

8. S. Perlmutter et al., Ap. J. 517, 565 (1999)

9. A.A. Starobinsky, Phys. Lett. B 91, 99 (1980)

10. A. Guth, Phys. Rev. D 23, 347 (1981)

11. A.R. Liddle, Phys. Lett. B 220, 502 (1989)

12. T. Charters, J.P. Mimoso, A. Nunes, Phys. Lett. B 472, 21 (2000)

13. J.D. Barrow, Phys. Rev. D 48, 1585 (1993)

14. J.D. Barrow, P. Saich, Class. Quantum Gravity 10, 279 (1993)

15. B. Ratra, P.J.E. Peebles, Phys. Rev. D 37, 3406 (1988)

16. J.D. Barrow, P. Saich, Class. Quantum Gravity 10, 279 (1993)

17. E.V. Linder, Phys. Rev. D 70, 023511 (2004)

18. P. Singh, M. Sani, N. Dadhich, Phys. Rev. D 68, 023522 (2003)
19. C. Armendariz-Picon, V. Mukhanov, P.J. Steinhardt, Phys. Rev. D 63, $103510(2001)$

20. T. Clifton, P.G. Ferreira, A. Padilla, C. Skordis, Phys. Rep. 513, 1 (2012)

21. S. Nojiri, S.D. Odintsov, V.K. Oikonomou, Phys. Rep. 692, 1 (2017)

22. T.P. Sotiriou, Gravity and scalar fields, in Modifications of Einstein's Theory of Gravity at Large Distances. Lecture Notes in Physics, vol. 892, ed. by E. Papantonopoulos (Springer, Cham, 2015)

23. C. Brans, R.H. Dicke, Phys. Rev. 124, 195 (1961)

24. J. O'Hanlon, Phys. Rev. Lett. 29, 137 (1972)

25. V. Faraoni, Cosmology in Scalar-Tensor Gravity, Fundamental Theories of Physics, vol. 139 (Kluwer Academic Press, Dordrecht, 2004)

26. G.W. Horndeski, Int. J. Ther. Phys. 10, 363 (1974)

27. C. Deffayet, G. Esposito-Farese, A. Vikman, Phys. Rev. D 79, 084003 (2009)

28. A. Nicolis, R. Rattazzi, E. Trincherini, Phys. Rev. D 79, 064036 (2009)

29. G. Leon, E.N. Saridakis, JCAP 03, 025 (2013)

30. N. Dimakis, A. Giacomini, S. Jamal, G. Leon, A. Paliathanasis, Phys. Rev. D 95, 064031 (2017)

31. R. De Arcia, T. Gonzalez, F.A. Horta-Rangel, G. Leon, U. Nucamendi, I. Quiros, Class. Quantum Gravity 35(14), 145001 (2018)

32. R. De Arcia, T. Gonzalez, G. Leon, U. Nucamendi, I. Quiros, Class. Quantum Gravity 33(12), 125036 (2016)

33. S. Bahamonde, K.F. Dialektopoulos, J. Levi Said, Phys. Rev. D 100(6), 064018 (2019)

34. S. Bahamonde, K.F. Dialektopoulos, V. Gakis, J. Levi Said, Phys. Rev. D 101(8), 084060 (2020)

35. S. Bahamonde, K.F. Dialektopoulos, M. Hohmann, J. Levi Said. arXiv:2003.11554 [gr-qc]

36. G. Kofinas, E.N. Saridakis, Phys. Rev. D 90, 084044 (2014)

37. G. Kofinas, G. Leon, E.N. Saridakis, Class. Quantum Gravity 31, 175011 (2014)

38. W. Hu, Phys. Rev. D 71, 047301 (2005)

39. Y.-F. Cai, E.N. Saridakis, M.R. Setare, J.-Q. Xia, Phys. Rep. 493, $1(2010)$

40. R. Lazkoz, G. Leon, Phys. Lett. B 638, 303-309 (2006)

41. R. Lazkoz, G. Leon, I. Quiros, Phys. Lett. B 649, 103 (2007)

42. G. Leon, Y. Leyva, J. Socorro, Phys. Lett. B 732, 285-297 (2014)

43. G. Leon, A. Paliathanasis, J.L. Morales-Martínez, Eur. Phys. J. C 78(9), 753 (2018)

44. D. Wands, Lect. Notes Phys. 738, 275 (2008)

45. P. Carrilho, D. Mulryne, J. Ronaye, T. Tenkanen, JCAP 06, 032 (2018)

46. P. Christodoulidis, D. Roest, E.I. Sfakianakis, JCAP 11, 002 (2019)

47. P.M. Sa, Triple unification of inflation, dark energy, and dark matter in two-scalar-field cosmology. arXiv:2007.07109

48. A. Arbey, Phys. Rev. D 74, 043516 (2006)

49. C.-J. Gao, Y.-G. Shen, Phys. Lett. B 541, 1 (2002)

50. D.S.M. Alves, G.M. Kremer, JCAP 10, 009 (2004)

51. L.P. Chimento, Phys. Rev. D 81, 043525 (2010)

52. S. Basilakos, G. Leon, G. Papagiannopoulos, E.N. Saridakis, Phys. Rev. D 100(4), 043524 (2019)

53. A. Alho, C. Uggla, J. Wainwright, JCAP 09, 045 (2019)

54. A. Alho, C. Uggla, J. Wainwright. arXiv:2006.00800 [gr-qc]

55. R.G. Landim, Eur. Phys. J. C 79(11), 889 (2019)

56. G.B. Zhao, J.Q. Xia, M. Li, B. Feng, X. Zhang, Phys. Rev. D 72, $123515(2005)$

57. D.I. Kaiser, E.I. Sfakianakis, Phys. Rev. Lett. 112(1), 011302 (2014)

58. A.A. Coley, R.J. van den Hoogen, Phys. Rev. D 62, 023517 (2000)

59. I.V. Fomin, J. Phys. Conf. Ser. 918, 012009 (2017)

60. S.V. Ketov, Quantum Non-linear Sigma Models (Springer, Berlin, 2000) 
61. A. Paliathanasis, G. Leon, S. Pan, Gen. Relat. Gravit. 51, 106 (2019)

62. S.V. Chervon, Quantum Matter 2, 71 (2013)

63. S.V. Chervon, I.V. Fomin, E.O. Pozdeeva, M. Sami, S.Y. Vernov, Phys. Rev. D 100(6), 063522 (2019)

64. A. Paliathanasis, M. Tsamparlis, Phys. Rev. D 90, 43529 (2014)

65. P. Christodoulakis, D. Roest, E.I. Sfakianakis, JCAP 19, 059 (2019)

66. A. Paliathanasis, Dynamics of chiral cosmology. arXiv:2003.05342

67. N. Dimakis, A. Paliathanasis, P.A. Terzis, T. Christodoulakis, EPJC 79, 618 (2019)
68. N. Dimakis, A. Paliathanasis, Crossing the phantom divide line as an effect of quantum transitions. arXiv:2001.09687

69. E.J. Copeland, M. Sami, S. Tsujikawa, IJMPD 15, 1753 (2006)

70. A. Halder, A. Paliathanasis, P.G.L. Leach, Symmetry 10, 744 (2018)

71. M. Tsamparlis, A. Paliathanasis, Symmetry 10, 233 (2018)

72. L. Karpathopoulos, M. Tsamparlis, A. Paliathanasis, J. Geom. Phys. 133, 279 (2018) 